\title{
Stability and convergence of the space fractional variable-order Schrödinger equation
}

\author{
Abdon Atangana ${ }^{1 *}$ and Alain $\mathrm{H} \mathrm{Cloot}^{2}$
}

\section{*Correspondence:}

abdonatangana@yahoo.fr

${ }^{1}$ Institute for Groundwater Studies,

Faculty of Natural and Agricultural

Sciences, University of the Free

State, Bloemfontein, South Africa

Full list of author information is

available at the end of the article

\begin{abstract}
The space fractional Schrödinger equation was further extended to the concept of space fractional variable-order derivative. The generalized equation is very difficult to handle analytically. We solved the generalized equation numerically via the Crank-Nicholson scheme. The stability and the convergence of the space fractional variable-order Schrödinger equation were presented in detail.
\end{abstract}

Keywords: Schrödinger equation; variable-order derivative; Crank-Nicholson scheme; convergence; stability

\section{Introduction}

In quantum mechanics, the Schrödinger equation is a partial differential equation that describes how the quantum state of some physical system changes with time. It was formulated in late 1925, and published in 1926, by the Austrian physicist Erwin Schrödinger. Fractional-order derivatives can be dated back to the seventeenth century [1]. It has primarily developed as a pure notional field of mathematics since its appearance. However, fractional-order differential systems have been proved to be useful in physics, engineering and even financial analysis in the last few decades [2]. The fractional-order dynamical systems consist of viscoelastic systems [3], dielectric polarization [4], electrode-electrolyte polarization [5], electromagnetic waves [6], quantitative finance [7] and quantum evolution of complex systems [8]. In particular, in fractional quantum evolution fields, there are many results obtained by some scholars (see in [8-17]). For instance, Laskin put up the space fractional quantum mechanics in 2000 [9-12], and the Schrödinger equation with space fractional derivative was also studied [13-16]. In the same way, the time fractional Schrödinger equation was also discussed in the works [15-17]. However, many researchers in the field of mathematics and physics paid attention to study physical problems described by the variable order derivative (see [18-22]). As a result of important differences between fractional order differential equations (FODE) and variable-order differential equations (VFODE), most characteristics or conclusions of the FODE systems can sometimes be extended to the case of the VFODE systems. Recently, many efforts have been devoted to the study of chaotic dynamics of variable-order differential systems [23-28]. Many current results about variable-order chaotic systems, however, are accomplished only by numerical simulations. In this work we further investigate the possibility of extension of the frac-

(c) 2013 Atangana and Cloot; licensee Springer. This is an Open Access article distributed under the terms of the Creative Commons Attribution License (http://creativecommons.org/licenses/by/2.0), which permits unrestricted use, distribution, and reproduction in any medium, provided the original work is properly cited. 
tional Schrödinger equation to the concept of variable-order time fractional. The stability and the convergence of the new equation will be investigated in detail.

\section{Problem formulation}

For the readers that are not acquainted with the concept of the variable-order derivative, we start this section and we present the basic definition of this derivative.

\subsection{Variable-order differential operator}

Let $f: \mathbb{R} \rightarrow \mathbb{R}, x \rightarrow f(x)$ denote a continuous and necessary differentiable, let $\alpha(x)$ be a continuous function in $(0,1]$. Then its variation-order differential is defined as:

$$
D_{0}^{\alpha(x)}(f(x))=\frac{1}{\Gamma(1-\alpha(x))} \int_{0}^{x}(x-t)^{-\alpha(t)} \frac{d f(t)}{d t} d t .
$$

The above derivative is called the Caputo variable-order differential operator; in addition, the derivative of the constant is zero.

\subsection{Modification of the Schrödinger equation}

The most famous example is the nonrelativistic Schrödinger equation for a single particle moving in an electric field as given below

$$
i \frac{h}{2 \pi} \frac{\partial \Psi(r, t)}{\partial t}=\left[-\frac{\left[\frac{h}{2 \pi}\right]^{2}}{2 m} \nabla^{2}+V(r, t)\right] \Psi(r, t),
$$

where $m$ is the particle's mass, $V$ is its potential energy, $\nabla^{2}$ is the Laplacian, and $\Psi$ is the wave-function, more precisely, in this context, it is called the position-space wavefunction. In plain language, it means total energy equals kinetic energy plus potential energy. The Schrödinger equation plays the role of Newton's law and conservation of energy in classical mechanics meaning it predicts the future behavior of a dynamic system. It is a wave equation in terms of the wave-function, which predicts analytically and precisely the probability of events or outcome. The detailed outcome is not strictly determined, but given a large number of events, the Schrödinger equation will predict the distribution of results. The kinetic and potential energies are transformed into the Hamiltonian which acts upon the wave-function to generate the evolution of the wave-function in time and space. The Schrödinger equation gives the quantized energies of the system and gives the form of the wave-function so that other properties may be calculated. This equation was extended to the concept of fractional order derivative with great success (see [15-17]). In this paper we extend this equation to the concept of fractional variable-order derivative by replacing the Laplace operator in Equation (2.1) by the variable-order Laplace operator to obtain:

$$
i \frac{h}{2 \pi} \frac{\partial \Psi(r, t)}{\partial t}=\left[-\frac{\left[\frac{h}{2 \pi}\right]^{2}}{2 m} \nabla^{\alpha(x, t)}+V(r, t)\right] \Psi(r, t), \quad 0<\alpha(x, t) \leq 2 .
$$

The above equation will be called the space fractional variable-order Schrödinger equation. This modified equation cannot be solved analytically, therefore, in the following section we present, the discussion underpinning the numerical solution via the CrankNicholson scheme. 


\section{Numerical solution of the modified equation}

Environmental phenomena, such as space fractional variable-order Schrödinger equation, are highly complex phenomena, which do not lend themselves readily to analysis of analytical models. The discussion presented in this section will therefore be devoted to the derivation of numerical solution to the space fractional variable-order Schrödinger equation (2.3).

Numerical methods yield approximate solutions to the governing equation through the discretization of space and time. Within the discretized problem domain, the variable internal properties, boundaries, and stresses of the system are approximated. Deterministic, distributed-parameter, numerical models can relax the rigid idealized conditions of analytical models or lumped-parameter models, and they can therefore be more realistic and flexible for simulating fields conditions. The finite difference schemes for constantorder time or space fractional diffusion equations have been extensively considered in the literature; see, for instance, the work done in [14-19]. For constant-order time fractional diffusion equations, an implicit difference approximation scheme was presented in [20]. The weighted average finite difference method was initiated in [21]. Podlubny proposed the matrix approach for fractional diffusion equations [22] and Hanert proposed a flexible numerical scheme for the discretization of the space-time fractional diffusion equation [23]. Recently, Zhuang well thought out the numerical schemes for the variableorder (VO) space fractional advection-dispersion equation [16]. Lin studied the explicit scheme for the $\mathrm{VO}$ nonlinear space fractional diffusion equation [24]. Recently, Atangana and Botha presented the stability and the convergence of the generalized time-fractional variable-order groundwater flow equation [27].

\subsection{Crank-Nicholson scheme}

Before performing the numerical methods, we assume Equation (2.3) has a unique and sufficiently smooth solution. To establish the numerical schemes for the above equation, we let $x_{l}=l h, 0 \leq l \leq M, M h=L, t_{k}=k \tau, 0 \leq k \leq N, N \tau=T, h$ is the step and $\tau$ is the time size, $M$ and $N$ are grid points.

We introduce the Crank-Nicholson scheme as follows. Firstly, the discretization of first and second-order space derivative is stated as

$$
\begin{aligned}
& \frac{\partial \Psi}{\partial t}=\frac{1}{2}\left(\left(\frac{\Psi\left(r_{l+1}, t_{k+1}\right)-\Psi\left(r_{l+1}, t_{k-1}\right)}{2(\tau)}\right)+\left(\frac{\Psi\left(r_{l}, t_{k+1}\right)-\Psi\left(r_{l}, t_{k-1}\right)}{2(\tau)}\right)\right)+O(\tau), \\
& V=\frac{1}{2}\left(V\left(r_{l}, t_{k+1}\right)+V\left(r_{l}, t_{k}\right)\right) .
\end{aligned}
$$

The Crank-Nicholson scheme for the VO space fractional Schrödinger equation can be stated as follows:

$$
\begin{aligned}
\frac{\partial^{\alpha_{l}^{k+1}} \Phi\left(r_{l}, t_{k+1}\right)}{\partial r_{l}^{\alpha_{l}^{k+1}}=} & \frac{h^{-\alpha_{l}^{k+1}}}{\Gamma\left(2-\alpha_{l}^{k+1}\right)}\left(\Psi\left(r_{l+1}, t_{k}\right)-\Psi\left(r_{l}, t_{k}\right)+\sum_{j=1}^{k}\left[\Psi\left(r_{l+1-j}, t_{k}\right)-\Psi\left(r_{l-j}, t_{k}\right)\right]\right. \\
& \left.\times\left[(j+1)^{1-\alpha_{l}^{k+1}}-(j)^{1-\alpha_{l}^{k+1}}\right]\right)+O(h) .
\end{aligned}
$$


It is important to point out that the quadrature formula (3.3) does not provide the values of the time fractional derivative at $r=0$ which are not required by the implicit finite difference and the Crank-Nicholson method schemes that follow.

Now, the Crank-Nicholson method with the discrete formulas (3.1), (3.2) and (3.4) is used to estimate the space variable-order fractional derivative to solve numerically the $\mathrm{VO}$ space fractional Schrödinger equation (2.3). If the potential is bounded from below, meaning there is a minimum value of potential energy, the eigen-functions of the Schrödinger equation have energy, which is also bounded from below. This can be seen most easily by using the variational principle. For simplicity, we assume that the potential energy $V$ has upper and lower boundaries, and we consider its average value $V(r, t)=\bar{V}$ in this paper. Using (3.1), (3.2) and (3.3) the restriction of the exact solution to the grid points centred at $\left(x_{l} ; t_{k}\right)=(l h ; \tau k)$; in Equation $(2.3)$, satisfies for $l=1,2, \ldots, N-1$ :

$$
\begin{aligned}
\frac{1}{2} i \frac{h}{2 \pi} & \left(\left(\frac{\Psi\left(r_{l+1}, t_{k+1}\right)-\Psi\left(r_{l+1}, t_{k-1}\right)}{2(\tau)}\right)+\left(\frac{\Psi\left(r_{l}, t_{k+1}\right)-\Psi\left(r_{l}, t_{k-1}\right)}{2(\tau)}\right)\right)+O(\tau) \\
& -\bar{V}\left[\frac{1}{2}\left(\Psi\left(r_{l}, t_{k+1}\right)+\Psi\left(r_{l}, t_{k}\right)\right)\right] \\
= & \frac{\left[\frac{h}{2 \pi}\right]^{2}}{2 m} \frac{h^{-\alpha_{l}^{k+1}}}{\Gamma\left(2-\alpha_{l}^{k+1}\right)}\left(\Psi\left(r_{l+1}, t_{k}\right)-\Psi\left(r_{l}, t_{k}\right)\right. \\
& +\sum_{j=1}^{k}\left[\Psi\left(r_{l+1-j}, t_{k}\right)-\Psi\left(r_{l-j}, t_{k}\right)\right]\left[(j+1)^{1-\alpha_{l}^{k+1}}-(j)^{\left.1-\alpha_{l}^{k+1}\right]}\right)+O(h), \\
\frac{1}{2} i \frac{h}{2 \pi} & \left(\left(\frac{\Psi\left(r_{l+1}, t_{k+1}\right)-\Psi\left(r_{l+1}, t_{k-1}\right)}{2(\tau)}\right)+\left(\frac{\Psi\left(r_{l}, t_{k+1}\right)-\Psi\left(r_{l}, t_{k-1}\right)}{2(\tau)}\right)\right) \\
& -\bar{V}\left[\frac{1}{2}\left(\Psi\left(r_{l}, t_{k+1}\right)+\Psi\left(r_{l}, t_{k}\right)\right)\right] \\
= & \frac{\left[\frac{h}{2 \pi}\right]^{2}}{2 m} \frac{h^{-\alpha_{l}^{k+1}}}{\Gamma\left(2-\alpha_{l}^{k+1}\right)}\left(\Psi\left(r_{l+1}, t_{k}\right)-\Psi\left(r_{l}, t_{k}\right)\right. \\
& +\sum_{j=1}^{k}\left[\Psi\left(r_{l+1-j}, t_{k}\right)-\Psi\left(r_{l-j}, t_{k}\right)\right]\left[(j+1)^{1-\alpha_{l}^{k+1}}-(j)^{\left.1-\alpha_{l}^{k+1}\right]}\right)+T(r, t),
\end{aligned}
$$

where $T(r, t)$ is the truncation term. Thus, according to Equation (3.4), the numerical method is consistent, first order correct in time and second order correct in space.

Now, for simplicity, we let $\Psi\left(r_{l}, t_{k}\right)=\Psi_{l}^{k}$ such that, the resulting finite difference equations are defined by

$$
\begin{aligned}
\frac{1}{2} i \frac{h}{2 \pi}\left(\left(\frac{\Psi_{l+1}^{k+1}-\Psi_{l+1}^{k-1}}{2(\tau)}\right)+\left(\frac{\Psi_{l}^{k+1}-\Psi_{l}^{k-1}}{2(\tau)}\right)\right)-\bar{V}\left[\frac{1}{2}\left(\Psi_{l}^{k+1}+\Psi_{l}^{k}\right)\right] \\
=\frac{\left[\frac{h}{2 \pi}\right]^{2}}{2 m} \frac{h^{-\alpha_{l}^{k+1}}}{\Gamma\left(2-\alpha_{l}^{k+1}\right)}\left(\Psi_{l+1}^{k}-\Psi_{l}^{k}\right. \\
\left.\quad+\sum_{j=1}^{k}\left[\Psi_{l+1-j}^{k}-\Psi_{l-j}^{k}\right]\left[(j+1)^{1-\alpha_{l}^{k+1}}-(j)^{1-\alpha_{l}^{k+1}}\right]\right) .
\end{aligned}
$$


Setting

$$
\begin{aligned}
& H_{l}^{k+1}=\left(\frac{\left[\frac{h}{2 \pi}\right]^{2}}{2 m}\right)^{-1} \frac{h^{1+\alpha_{l}^{k+1}} i \Gamma\left(2-\alpha_{l}^{k+1}\right)}{8 \pi \tau} ; \\
& V_{l}^{k+1}=\frac{-\bar{V} h^{\alpha_{l}^{k+1}} \Gamma\left(2-\alpha_{l}^{k+1}\right)}{2}\left(\frac{\left[\frac{h}{2 \pi}\right]^{2}}{2 m}\right)^{-1}, \\
& b_{j}^{l, k+1}=(j+1)^{1-\alpha_{l}^{k+1}}-(j)^{1-\alpha_{l}^{k+1}} \text { and } \lambda_{j}^{l, k+1}=b_{j-1}^{l, k+1}-b_{j}^{l, k+1} .
\end{aligned}
$$

Substituting (3.6) into (3.5) and reordering, we obtained the following:

$$
\begin{aligned}
& H_{l}^{k+1}\left(\left(\Psi_{l+1}^{k+1}-\Psi_{l+1}^{k-1}\right)+\left(\Psi_{l}^{k+1}-\Psi_{l}^{k-1}\right)\right)+V_{l}^{k+1}\left[\left(\Psi_{l}^{k+1}+\Psi_{l}^{k}\right)\right] \\
& \quad=\left(\Psi_{l+1}^{k}-\Psi_{l}^{k}+\sum_{j=1}^{k}\left[\Psi_{l+1-j}^{k}-\Psi_{l-j}^{k}\right] b_{j}^{l, k+1}\right) .
\end{aligned}
$$

With the inclusion of the boundary conditions: $\Psi_{0}^{k}=\Psi_{N}^{k}=0, n=1,2, \ldots, N-1$. It is important to note that, Equation (3.7) requires, at each time step, to solve a tri-diagonal system of linear equations where the right-hand side utilizes all the history of the computed solution up to that time.

Our next concern here is to show that the stability of the fractional numerical schemes can be analyzed very successfully with no trouble and powerfully with the distinguished Von Neumann method of non-fractional partial differential equations [25].

\section{Stability analysis of space fractional variable-order Schrödinger equation}

In this section, we will analyze the stability conditions of the Crank-Nicholson scheme for the space fractional variable-order Schrödinger equation.

Let $\zeta_{l}^{k}=\Psi_{l}^{k}-\mathrm{X}_{l}^{k}$, here $\mathrm{X}_{l}^{k}$ is the approximate solution at the point $\left(x_{l}, t_{k}\right)(k=1,2, \ldots, N$, $l=1,2, \ldots, M-1)$ and in addition $\zeta^{k}=\left[\zeta_{1}^{k}, \zeta_{2}^{k}, \ldots, \zeta_{M-1}^{k}\right]^{T}$ and the function $\zeta^{k}(x)$ is chosen to be:

$$
\zeta^{k}(x)= \begin{cases}\zeta_{l}^{k} & \text { if } x_{l}-\frac{h}{2}<x \leq x_{l}+\frac{h}{2}, l=1,2, \ldots, M-1 \\ 0 & \text { if } L-\frac{h}{2}<x \leq L\end{cases}
$$

Then, the function $\zeta^{k}(x)$ can be expressed in Fourier series as follows:

$$
\begin{aligned}
& \zeta^{k}(x)=\sum_{m=-\infty}^{m=\infty} \delta_{m}(m) \exp [2 i \pi m k / L] \\
& \delta_{k}(x)=\frac{1}{L} \int_{0}^{L} \rho^{k}(x) \exp \left[\frac{2 i \pi m x}{L}\right] d x .
\end{aligned}
$$

It was established by [20] that

$$
\left\|\rho^{2}\right\|_{2}^{2}=\sum_{m=-\infty}^{m=\infty}\left\|\delta_{k}(m)\right\|^{2}
$$

Observe that for all $k, l \geq 1,0 \leq 1-\alpha_{l}^{k+1}<1$. In addition, according to the problem under investigation, the Planck constant $h, m$ the particle's mass and $V$ its potential energy are 
positive constants. Then the following properties of the coefficients $H_{l}^{k+1}, V_{l}^{k+1}, \lambda_{j}^{l, k+1}$ and $b_{l}^{k+1}$ can be established:

1. $-V_{l}^{k+1}, H_{l}^{k+1}$ are positive for all $l=1,2, \ldots, M-1$,

2. $0<\lambda_{j}^{l, k} \leq \lambda_{j-1}^{l, k} \leq 1$ for all $l=1,2, \ldots, M-1$,

3. $0 \leq b_{j}^{l, k} \leq 1, \sum_{j=0}^{k-1} b_{j+1}^{l, k+1}=1-\lambda_{k}^{l, k+1}$ for all $l=1,2, \ldots, M-1$.

Note that in this case, the error committed while approximating the solution of the space fractional variable-order Schrödinger equation with the Crank-Nicholson scheme can be presented as follows:

$$
\begin{aligned}
& H_{l}^{k+1}\left(\left(\zeta_{l+1}^{k+1}-\zeta_{l+1}^{k-1}\right)+\left(\zeta_{l}^{k+1}-\zeta_{l}^{k-1}\right)\right)+V_{l}^{k+1}\left[\left(\zeta_{l}^{k+1}+\zeta_{l}^{k}\right)\right] \\
& =\left(\zeta_{l+1}^{k}-\zeta_{l}^{k}+\sum_{j=1}^{k}\left[\zeta_{l+1-j}^{k}-\zeta_{l-j}^{k}\right] b_{j}^{l, k+1}\right) .
\end{aligned}
$$

If we assume that: $\zeta_{l}^{k}$ in Equation (4.11) can be put in the delta-exponential form as follows:

$$
\zeta_{l}^{k}=\delta_{k} \exp [i \varphi l k]
$$

where $\varphi$ is a real spatial wave number, new replacing the above Equation (4.6) in (4.5) we obtain the following expression:

$$
\begin{aligned}
& \delta_{k+1}\left(2 H_{l}^{1+k} \sin ^{2}\left(\frac{\varphi h}{2}\right)+2 V_{l}^{1+k} \sin ^{2}\left(\frac{\varphi h}{2}\right)\right) \\
& =\left[-1-2 V_{l}^{1+k} \sin ^{2}\left(\frac{\varphi h}{2}\right)-\lambda_{l}^{l, k+1}\right] \delta_{k}+\sum_{j=0}^{k-1} \lambda_{j+1}^{l, k+1} \delta_{k-j}+\lambda_{k}^{l, k+1} \delta_{0} \quad \text { for } k \geq 1, \\
& \delta_{1}\left(2 H_{l}^{1} \sin ^{2}\left(\frac{\varphi h}{2}\right)+2 V_{l}^{1} \sin ^{2}\left(\frac{\varphi h}{2}\right)\right)=\left[-1-2 V_{l}^{1} \sin ^{2}\left(\frac{\varphi h}{2}\right)\right] \delta_{0} \quad \text { for } k=0 .
\end{aligned}
$$

We next show that, for all $k=1,2, \ldots, N-1$ the solution of Equation (4.7) complies with the following condition:

$$
\left|\delta_{k}\right|<\left|\delta_{0}\right|
$$

To achieve this, we make use of the recurrence technique on the natural number $k$.

For $k=1$ and $-V_{l}^{k+1}, H_{l}^{k+1}$ are positive for all $l=1,2, \ldots, M-1$, then we obtain:

$$
\frac{\left|\delta_{1}\right|}{\left|\delta_{0}\right|}=\left|\frac{\left[1+2 V_{l}^{1} \sin ^{2}\left(\frac{\varphi h}{2}\right)\right]}{\left[2 H_{l}^{1} \sin ^{2}\left(\frac{\varphi h}{2}\right)+2 V_{l}^{1} \sin ^{2}\left(\frac{\varphi h}{2}\right)\right]}\right|<1 .
$$

The condition is true for $k=0$.

Assuming that for $m=2,3, \ldots, k$ the property is verified. Then

$$
\left|\delta_{k+1}\right|=\left|\frac{\left[-1-2 V_{l}^{1+k} \sin ^{2}\left(\frac{\varphi h}{2}\right)-\lambda_{1}^{l, k+1}\right] \delta_{k}+\sum_{j=0}^{k-1} \lambda_{j+1}^{l, k+1} \delta_{k-j}+\lambda_{k}^{l, k+1} \delta_{0}}{2 H_{l}^{1+k} \sin ^{2}\left(\frac{\varphi h}{2}\right)+2 V_{l}^{1+k} \sin ^{2}\left(\frac{\varphi h}{2}\right)}\right| .
$$


Using the triangular inequality we obtain:

$$
\begin{aligned}
&\left|\delta_{k+1}\right| \leq \frac{\left|-1-2 V_{l}^{1+k} \sin ^{2}\left(\frac{\varphi h}{2}\right)-\lambda_{1}^{l, k+1}\right|\left|\delta_{k}\right|+\sum_{j=0}^{k-1}\left|\lambda_{j+1}^{l, k+1}\right|\left|\delta_{k-j}\right|+\left|\lambda_{k}^{l, k+1}\right|\left|\delta_{0}\right|}{\left|2 H_{l}^{1+k} \sin ^{2}\left(\frac{\varphi h}{2}\right)+2 V_{l}^{1+k} \sin ^{2}\left(\frac{\varphi h}{2}\right)\right|}, \\
&\left|\delta_{k+1}\right| \leq \frac{\left|1+2 V_{l}^{1+k} \sin ^{2}\left(\frac{\varphi h}{2}\right)+\sum_{j=0}^{k-1}\right| \lambda_{j+1}^{l, k+1}||\left|\delta_{0}\right|}{\left|2 H_{l}^{1+k} \sin ^{2}\left(\frac{\varphi h}{2}\right)+2 V_{l}^{1+k} \sin ^{2}\left(\frac{\varphi h}{2}\right)\right|}, \\
&\left|\delta_{k+1}\right| \leq \frac{\left|2 H_{l}^{1+k} \sin ^{2}\left(\frac{\varphi h}{2}\right)+2 V_{l}^{1+k} \sin ^{2}\left(\frac{\varphi h}{2}\right)\right|\left|\delta_{0}\right|}{\left|2 H_{l}^{1+k} \sin ^{2}\left(\frac{\varphi h}{2}\right)+2 V_{l}^{1+k} \sin ^{2}\left(\frac{\varphi h}{2}\right)\right|} \\
&\left|\delta_{k+1}\right| \leq\left|\delta_{0}\right|
\end{aligned}
$$

and the proof is completed.

\section{Convergence analysis of space fractional variable-order Schrödinger equation}

Assuming that, $\Psi\left(r_{l}, t_{k}\right)(l=1,2, \ldots, M, k=1,2, \ldots, N-1)$ is the exact solution of our problem at the point $\left(r_{l}, t_{k}\right)$, by letting $\Omega_{l}^{k}=\Psi\left(r_{l}, t_{k}\right)-\mathrm{X}_{l}^{k}$ and $\Omega^{k}=\left(0, \Omega_{1}^{k}, \Omega_{2}^{k}, \ldots, \Omega_{M-1}^{k}\right)$ substituting this in Equation (4.5), we obtain:

$$
\begin{aligned}
& \Omega_{l}^{1}\left(H_{l}^{1}+V_{l}^{1}\right)+\Omega_{l+1}^{1}\left(H_{l}^{1}\right)=T_{l}^{1} \quad \text { for } k=0, \\
& \Omega_{l}^{k+1}\left(H_{l}^{k+1}+V_{l}^{k+1}\right)+\Omega_{l+1}^{k+1} H_{l}^{k+1}=T_{l}^{k+1} \quad \text { for } k \geq 1,
\end{aligned}
$$

here

$$
T_{l}^{k+1}=\frac{1}{2} i \frac{h}{2 \pi}\left(\left(\frac{\Psi\left(r_{l+1}, t_{k+1}\right)}{2(\tau)}\right)+\left(\frac{\Psi\left(r_{l}, t_{k+1}\right)}{2(\tau)}\right)\right)-\bar{V}\left[\frac{1}{2}\left(\Psi\left(r_{l}, t_{k+1}\right)\right)\right] .
$$

From Equations (3.1) and (3.2) we have the following:

$$
\begin{aligned}
& \frac{\partial \Psi}{\partial t}+V \tau=\frac{1}{2}\left(\left(\frac{\Psi\left(r_{l+1}, t_{k+1}\right)-\Psi\left(r_{l+1}, t_{k-1}\right)}{2(\tau)}\right)+\left(\frac{\Psi\left(r_{l}, t_{k+1}\right)-\Psi\left(r_{l}, t_{k-1}\right)}{2(\tau)}\right)\right), \\
& \frac{\partial^{\alpha_{l}^{k+1}} \Phi\left(r_{l}, t_{k+1}\right)}{\partial r_{l}^{\alpha_{l}^{+1}}}+w h \\
& \quad=\frac{h^{-\alpha_{l}^{k+1}}}{\Gamma\left(2-\alpha_{l}^{k+1}\right)}\left(\Psi\left(r_{l+1}, t_{k}\right)-\Psi\left(r_{l}, t_{k}\right)+\sum_{j=0}^{k} \lambda_{j+1}^{l, k+1} \Psi\left(r_{l-j}, t_{k}\right)-b_{j}^{l, k+1} \Psi\left(r_{l}, t_{0}\right)\right) .
\end{aligned}
$$

From the above we have that:

$$
T_{l}^{k+1} \leq K\left(h^{1+\alpha_{l}^{k+1}}+\tau h^{\alpha_{l}^{k}}\right)
$$

where $V, w$ and $K$ are constants. Taking into account the Caputo type fractional derivative, the detailed error analysis on the above schemes can refer to the work in [25] and further work in [26]. 
Lemma $1\left\|\Omega^{k+1}\right\|_{\infty} \leq K\left(h^{1+\alpha_{l}^{k+1}}+\tau h^{\alpha_{l}^{k}}\right)\left(\Omega_{j}^{l, k+1}\right)^{-1}$ is true for $k=0,1,2, \ldots, N-1$ where $\left\|w^{k}\right\|_{\infty}=\max _{1 \leq l \leq M-1}\left(\Omega^{k}\right), K$ is a constant. In addition,

$$
\alpha^{k+1}= \begin{cases}\min _{1 \leq l \leq M-1} \alpha_{l}^{k+1}, & \text { if } \tau<1, \\ \max _{1 \leq l \leq M-1} \alpha_{l}^{k+1}, & \text { if } \tau>1 .\end{cases}
$$

This can be achieved via the recurrence technique on the natural number $k$. When $k=0$, we have the following:

$$
\left|\Omega_{l}^{1}\right| \leq\left|\Omega_{l}^{1}\right|\left(H_{l}^{1}+V_{l}^{1}\right)+\left|\Omega_{l+1}^{1}\right|\left(H_{l}^{1}\right)=\left|T_{l}^{1}\right| \leq K\left(h^{1+\alpha_{l}^{k+1}}+\tau h^{\alpha_{l}^{k}}\right)\left(\Omega_{j}^{l, k+1}\right)^{-1} .
$$

Now suppose that $\left\|\Omega^{i+1}\right\|_{\infty} \leq K\left(\tau^{1+\alpha_{l}^{i+1}}+h^{2} \tau^{\alpha_{l}^{i}}\right)\left(\lambda_{j}^{l, i+1}\right)^{-1}, i=1, \ldots, N-2$. Then

$$
\begin{aligned}
& \left|\Omega_{l}^{k+1}\right| \leq\left|\Omega_{l}^{k+1}\left(H_{l}^{k+1}+V_{l}^{k+1}\right)+\Omega_{l+1}^{k+1} H_{l}^{k+1}-\sum_{j=0}^{k-1} \lambda_{j+1}^{l, k+1} \Omega_{k-j}-\lambda_{k}^{l, k+1} \Omega_{0}\right| \\
& \left|\Omega_{l}^{k+1}\right| \leq\left|\Omega_{l}^{k+1}\right|\left(H_{l}^{k+1}+V_{l}^{k+1}\right)+\left|\Omega_{l+1}^{k+1}\right| H_{l}^{k+1}+\sum_{j=0}^{k-1} \lambda_{j+1}^{l, k+1}\left|\Omega_{k-j}\right|+\lambda_{k}^{l, k+1}\left|\Omega_{0}\right| .
\end{aligned}
$$

Using the recurrence hypothesis, the above equation is reduced to the following expression:

$$
\begin{aligned}
& \left|\Omega_{l}^{k+1}\right| \leq\left|T_{l}^{k+1}+\sum_{j=0}^{k-1} \lambda_{j+1}^{l, k+1} \Omega_{k-j}\right|, \\
& \left|\Omega_{l}^{k+1}\right| \leq\left|T_{l}^{k+1}\right|+\sum_{j=0}^{k-1} \lambda_{j+1}^{l, k+1}\left|\Omega_{k-j}\right|, \\
& \left|\Omega_{l}^{k+1}\right| \leq K\left(\tau^{1+\alpha_{l}^{i+1}}+h^{2} \tau^{\alpha_{l}^{i}}\right)+\sum_{j=0}^{k-1} \lambda_{j+1}^{l, k+1}\left\|\Omega_{k-j}\right\|_{\infty}, \\
& \left|\Omega_{l}^{k+1}\right| \leq K\left(\tau^{1+\alpha_{l}^{i+1}}+h^{2} \tau^{\alpha_{l}^{i}}\right)\left(\lambda_{j}^{l, k+1}+\lambda_{0}^{l, k+1}-\lambda_{j}^{l, k+1}\right)\left(\lambda_{j}^{l, k+1}\right)^{-1}, \\
& \left|\Omega_{l}^{k+1}\right| \leq K\left(\tau^{1+\alpha_{l}^{i+1}}+h^{2} \tau^{\alpha^{i}}\right)\left(\lambda_{0}^{l, k+1}\right)\left(\lambda_{j}^{l, k+1}\right)^{-1}, \\
& \left|\Omega_{l}^{k+1}\right| \leq K\left(\tau^{1+\alpha_{l}^{i+1}}+h^{2} \tau^{\alpha_{l}^{i}}\right)\left(\lambda_{j}^{l, k+1}\right)^{-1},
\end{aligned}
$$

and the proof is completed.

Theorem 1 The Crank-Nicholson scheme of the space fractional variable-order Schrödinger equation is convergent, and there exists a positive constant $K$ such that:

$$
\left|\mathrm{X}_{l}^{k}-\Psi\left(x_{l}, t_{k}\right)\right| \leq K\left(\tau+h^{2}\right), \quad l=1,2, \ldots, M-1, k=1,2, \ldots, N .
$$

An interested reader can find the solvability of the Crank-Nicholson scheme in the work done in [24]. Therefore, the details of the proof will not be presented in this paper. 


\section{Conclusion}

We paid attention to study a possible generalization of the Schrödinger equation to the concept of space fractional variable-order derivative. The Laplace operator in the Schrödinger equation was replaced by the fractional variable Laplace operator. Since the new equation cannot be solved analytically, it was solved numerically via the CrankNicholson technique.

We presented in detail the stability and the convergence of this problem.

\section{Competing interests}

The authors declare that they have no competing interests.

\section{Authors' contributions}

AA wrote the first draft and AHC corrected and improved the final version. All authors read and approved the final draft.

\section{Author details}

${ }^{1}$ Institute for Groundwater Studies, Faculty of Natural and Agricultural Sciences, University of the Free State, Bloemfontein, South Africa. ${ }^{2}$ Department of Mathematics and Applied Mathematics, University of the Free State, Bloemfontein, South Africa.

\section{Acknowledgements}

Authors would like to thank the editor for his valuable time spared to access this manuscript and for his valuable comments toward the enhancement of this paper. Also, the authors would like to thank the anonymous referee for their valuable comments and suggestions toward the enhancement of this manuscript.

Received: 22 February 2013 Accepted: 20 March 2013 Published: 28 March 2013

\section{References}

1. Podlubny, I: Fractional Differential Equations. Academic Press, New York (1999)

2. Atangana, A, Secer, A: The time-fractional coupled-Korteweg-de-Vries equations. Abstr. Appl. Anal. 2013, Article ID 947986 (2013). doi:10.1155/2013/947986

3. Bagley, R, Calico, R: Fractional order state equations for the control of visco-elastically damped structures. J. Guid. Control Dyn. 14, 304-311 (1991)

4. Sun, H, Abdelwahed, A, Onaral, B: Linear approximation for transfer functions with a pole of fractional order. IEEE Trans. Autom. Control 29, 441-444 (1984)

5. Ichise, M, Nagayanagi, Y, Kojima, T: An analog simulation of non-integer order transfer functions for analysis of electrode process. J. Electroanal. Chem. Interfacial Electrochem. 33, 253-265 (1971)

6. Heaviside, O: Electromagnetic Theory. Chelsea, New York (1971)

7. Laskin, N: Fractional market dynamics. Physica A 287, 482-492 (2000)

8. Kunsezov, D, Bulagc, A, Dang, G: Quantum Lévy processes and fractional kinetics. Phys. Rev. Lett. 2,1136-1139 (1999)

9. Laskin, N: Fractional quantum mechanics. Phys. Rev. E 62, 3135-3145 (2000)

10. Laskin, N: Fractional quantum mechanics and Lévy path integrals. Phys. Lett. A 298, $298-305$ (2000)

11. Laskin, N: Fractals and quantum mechanics. Chaos 10, 780-790 (2000)

12. Laskin, N: Fractional Schrödinger equation. Phys. Rev. E 66, 056108 (2002)

13. Guo, X, Xu, M: Some physical applications of fractional Schrödinger equation. J. Math. Phys. 47, 82104 (2006)

14. Dong, J, Xu, M: Solutions to the space fractional Schrödinger equation using momentum representation method. J. Math. Phys. 48, 072105 (2007)

15. Wang, S, Xu, M: Generalized fractional Schrödinger equation with space-time fractional derivatives. J. Math. Phys. 48, $043502(2007)$

16. Dong, J, Xu, M: Space-time fractional Schrödinger equation with time-independent potentials. J. Math. Anal. Appl. 344, 1005-1017 (2008)

17. Naker, M: Time fractional Schrödinger equation. J. Math. Phys. 45(8), 3339-3352 (2004)

18. Umarov, S, Steinberg, S: Variable order differential equations and diffusion with changing modes. Z. Anal. Anwend. $28,431-450$ (2009)

19. Sun, HG, Chen, W, Chen, YQ: Variable order fractional differential operators in anomalous diffusion modeling. Physica A 388, 4586-4592 (2009)

20. Samko, SG, Ross, B: Integration and differentiation to a variable fractional order. Integral Transforms Spec. Funct. 1, 277-300 (1993)

21. Pedro, HTC, Kobayashi, MH, Pereira, JMC, Coimbra, CFM: Variable order modeling of diffusive-convective effects on the oscillatory flow past a sphere. J. Vib. Control 14, 1659-1672 (2008)

22. Solomon, TH, Weeks, ER, Swinney, HL: Observation of anomalous diffusion and Lévy flights in a two-dimensional rotating flow. Phys. Rev. Lett. 71, 3975-3978 (1993)

23. Zhuang, $P, L i u, F, A n h, V$, Turner, I: Numerical methods for the variable-order fractional advection-diffusion equation with a nonlinear source term. SIAM J. Numer. Anal. 47, 1760-1781 (2009)

24. Lin, R, Liu, F, Anh, V, Turner, I: Stability and convergence of a new explicit finite-difference approximation for the variable-order nonlinear fractional diffusion equation. Appl. Math. Comput. 212, 435-445 (2009)

25. Yuste, $S B, A c e d o, L$ : An explicit finite difference method and a new Von Neumann-type stability analysis for fractional diffusion equations. SIAM J. Numer. Anal. 42, 1862-1874 (2005) 
26. Lin, Y, Xu, C: Finite difference/spectral approximations for the time-fractional diffusion equation. J. Comput. Phys. 225, 1533-1552 (2007)

27. Atangana, A, Botha, JF: Generalized groundwater flow equation using the concept of variable order derivative. Bound. Value Probl. 2013, 53 (2013). doi:10.1186/1687-2770-2013-53

28. Atangana, A, Kilıçman, A: A possible generalization of acoustic wave equation using the concept of perturbed derivative order. Math. Probl. Eng. 2013, Article ID 696597 (2013). doi:10.1155/2013/696597

doi:10.1186/1687-1847-2013-80

Cite this article as: Atangana and Cloot: Stability and convergence of the space fractional variable-order Schrödinger equation. Advances in Difference Equations 2013 2013:80

Submit your manuscript to a SpringerOpen ${ }^{\circ}$ journal and benefit from:

- Convenient online submission

- Rigorous peer review

Immediate publication on acceptance

Open access: articles freely available online

- High visibility within the field

- Retaining the copyright to your article

Submit your next manuscript at $\boldsymbol{s p r i n g e r o p e n . c o m ~}$ 